FORMATION Formation emploi

Revue française de sciences sociales

107 | juillet-septembre 2009

La formation professionnelle en Amérique latine

\title{
L'enseignement professionnel en Argentine. Entre volontarisme et isolement dans la recherche d'un pays possible
}

Professional education in Argentina: finding a middle ground between

volontarism and isolation

Berufsbildung in Argentinien : Zwischen Voluntarismus und Isolierung im

Streben nach einem möglichen Land

La enseñanza profesional en La Argentina : Entre el voluntarismo y el

aislamiento en la búsqueda de un país posible

\section{Martín Spinosa et Julio Testa}

Traducteur : Mireille Zangani

\section{OpenEdition}

\section{Édition électronique}

URL : http://journals.openedition.org/formationemploi/1974

DOI : 10.4000/formationemploi.1974

ISSN : 2107-0946

\section{Éditeur}

La Documentation française

Édition imprimée

Date de publication : 1 septembre 2009

Pagination : 9-24

ISSN : 0759-6340

Référence électronique

Martín Spinosa et Julio Testa, « L'enseignement professionnel en Argentine. Entre volontarisme et isolement dans la recherche d'un pays possible », Formation emploi [En ligne], 107 | juillet-septembre 2009, mis en ligne le 01 septembre 2011, consulté le 30 octobre 2020. URL : http:// journals.openedition.org/formationemploi/1974 ; DOI : https://doi.org/10.4000/formationemploi.1974

() Tous droits réservés 


\section{DOSSIER}

\section{L'enseignement professionnel en Argentine. Entre volontarisme et isolement dans la recherche d'un pays possible'}

Par Martín Spinosa et Julio Testa*

L'article se propose de revisiter la notion de formation professionnelle et sa construction historique en Argentine. Il vise à dépasser un discours répandu qui fait reposer sur les organisations éducatives et les individus la responsabilité de former la force de travail.

La formation professionnelle constitue une des actions qui actuellement se diffuse très largement au sein des politiques publiques. Elle semble associée à la solution de problèmes sociaux multiples (comme l'abandon ou l'échec scolaire, le chômage, la difficulté à insérer les générations jeunes ou les groupes marginaux, le manque de productivité...) et on la considère, dans la majorité des travaux, comme une activité distincte du système éducatif et qui a un impact essentiel sur les individus aux prises avec les problèmes évoqués.

Cette perspective tend à naturaliser les propositions des politiques émanant des organismes multilatéraux de crédit, durant la décennie 90, à partir d'une analyse réductionniste de la relation entre éducation

\footnotetext{
${ }^{1}$ Nous souhaitons remercier Sébastian Otero pour la relecture
} attentive de ce travail et les apports réalisés.
* Martín A. Spinosa est pédagogue, spécialisé en sciences du travail, notamment en formation des adultes, chercheur au CEIL-PIETTE (Centro de Estudios y Investigaciones Laborales - Programa de Investigaciones Economicas sobre Technologia, Trabajo y Empleo) du CONICET (Consejo Nacional de Investigaciones Científicas y Técnicas) au département Éducation et Travail et professeur des Universités de Luján et Buenos Aires. II est spécialiste des questions d'éducation et de travail, notamment la construction des savoirs au travail en lien avec les savoirs scolaires. II a publié récemment: Spinosa M. (2009), "La construcción de la relación entre trabajo y saber. Las contribuciones de la corriente socio-histórica al análisis del trabajo », en: Feld V. y Eslava-CoboJ. Comps. ¿̇Hacia dónde va la neuropsicología? La perspectiva histórico cultural de Vigostsky y la neuropsicología. NOVEDUC. Buenos Aires. Spinosa. M., Drolas A. (2009), "De la certificaciónformación, a la certificación-aprendizaje en el proceso de integración del Mercosur», Calificaciones y Empleo 
n 62, Céreq. Delfini M., Spinosa M. (2008), Trabajo Argentino. Cambios y continuidades en 25 años de democracia. Colección " 25 años 25 libros», Biblioteca Nacional / UNGS.

Julio C. Testa est sociologue, chercheur au CEIL-PIETTE du CONICET et professeur des Universités de Luján et Buenos Aires. Il est le coordinateur du département Éducation et Travail. Spécialiste de l'éducation et du travail, il a réalisé de nombreux travaux d'enquêtes et il a coordonné de nombreuses équipes de recherche d'organismes publics nationaux et internationaux. II a publié récemment : Testa J. (Coord) (2008/9), La inserción profesional de los graduados de las carreras de sociología, trabajo social, relaciones de trabajo, ciencias políticas y ciencias de la comunicación de la Facultad de Ciencias Sociales de la UBA. UBA. Testa J. (Coord) (2005), "Cambio tecnológico y transformación de los perfiles profesionales de los técnicos químicos " Serie Informes de investigación n 14 , vol. 1-2 y 3-Ceil-Piette del Conicet.

et travail, et d'une méconnaissance de l'histoire de la formation de la force de travail en Argentine.

À cet égard, il nous semble nécessaire d'entamer cette réflexion par quelques précisions sur la construction historique de ce que, aujourd'hui, on appelle la formation professionnelle, pour ensuite mettre en évidence les tensions qui s'expriment actuellement sur la formation de la force de travail dans le cadre des débats pour un modèle de pays.

L'analyse historique de long terme est nécessaire pour au moins deux raisons :

a) en premier lieu, en raison de la nécessité théorique de distinguer, en termes généraux, les activités de production de la société dans ses diverses dimensions et la façon particulière dont ces actions s'exercent dans chaque contexte historique et géographique. Ainsi, bien que l'éducation en général se soit chargée d'assurer l'intégration des nouveaux membres dans la société, la séparation entre espaces de travail et vie privée est le fruit d'un lent processus dans l'histoire des sociétés occidentales, qui a affecté inégalement les différentes classes et secteurs à chaque période (Carton, 1985).

b) en second lieu, et déjà pour des raisons particulières liées à la réalité argentine, corréler la production et la transmission du savoir sur le travail à la formation professionnelle, comme on a l'habitude de le faire, pourrait nous conduire à conclure que dans notre pays, une telle chose n'existe pas ou bien seulement de façon marginale, nous amenant à considérer que la formation professionnelle est la source de tous les maux ou, ce qui revient au même, la solution à tous les problèmes et, en définitive, à nier l'existence de formes réelles de production et de circulation du savoir au travail.

L'analyse de la relation éducation-travail exige, d'une part, la construction d'une perspective ample, qui intègre dans son approche les divers niveaux d'analyse dans lesquels s'exprime cette relation :

a) structurelle, dans le sens ou les politiques et régimes d'accumulation du capitalisme national (agro exportateur ; industrialiste ; financier selon des époques) lui donnent de la substance ;

b) organisationnelle, en prenant en compte les diverses formes de fonctionnement et d'articulation des organisations éducatives et productives ;

c) subjective, dans la mesure où c'est dans les trajectoires particulières des individus et leurs apprentissages que se réalise finalement l'articulation.

D'autre part, la prise en compte des différentes dimensions qui entrent en jeu (économique, politique, éducationnelle et cognitive) dans les articulations qui se construisent socialement entre la production et la formation.

\section{UN SYSTÈME ÉDUCATIF EN TENSION (1880-1990)}

L'histoire du système éducatif argentin jusqu'à nos jours résulte de tensions entre une volonté «illuministe », selon le sens donné par Th. Adorno, ${ }^{2}$ de création d'un pays nouveau, et les structures économiques et productives propres aux modèles d'accumulation capitaliste qui se sont succédés.

\footnotetext{
${ }^{2}$ Le programme de l'Illustration prétendait dissoudre les mythes et en finir avec l'imagination à travers la science (AdornoHorkheimer, 1994: Adorno Theodor y Horkheimer Max, Dialéctica de la Ilustración, Trotta, Madrid, 1994. La Dialectique de la raison. Fragments philosophiques, Theodor W. Adorno, Max Horkheimer (Gallimard, 1974 et « Tel » n 82, 1983).
} 
Nous tenterons de présenter sommairement les principaux moments clés de ces tensions afin de mettre en lumière la genèse des contradictions liées à cette problématique.

\section{Une nation éducatrice (1880-1940)}

La préoccupation pour la formation (et la conformation) de la force de travail remonte, dans notre pays, aux origines mêmes de la nation indépendante. Héritières de la tradition révolutionnaire du $18^{\text {e }}$ siècle, les idées de Manuel Belgrano ${ }^{3}$ sur les finalités du Virreinato del Rio de La Plata (nom de la région pendant la colonisation espagnole), donnèrent naissance à la première école de Commerce. Au début du 19 e siècle déjà, et postérieurement à l'organisation institutionnelle du pays, les débats entamés au Congrès pédagogique de 1882 et, avant déjà, les écrits d'Alberdi et de Sarmiento ${ }^{4}$, mettaient en avant la nécessité de lier l'organisation de l'éducation nationale au développement productif d'un pays à construire. L'Argentine, à cette époque-là, était un pays agro-exportateur dont le marché interne dépendait de la production locale de biens plus ou moins artisanaux et de l'importation de produits manufacturés, principalement d'Angleterre.

La loi 1420 d’Éducation Commune, promulguée en 1884, va impulser un système éducatif commun, graduel, laïque et obligatoire pour l'ensemble de la population jusqu'à l'âge de 14 ans, dont le principal objectif est la construction d'une conscience nationale, destinée à la population immigrante.

L'enseignement des arts et métiers fut exclu de cette organisation du système éducatif et le développement industriel naissant ne requérait pas plus de force de travail qualifiée que celle susceptible d'être apportée par les immigrés européens. Italiens, espagnols, et dans une moindre mesure, portugais, polonais, allemands (entre autres pays qui furent

\footnotetext{
${ }^{3}$ Manuel Belgrano était fonctionnaire du Virreinato ; il participa ensuite activement à la révolution de Mai 1810 comme militaire et homme politique. Il est considéré comme un des héros nationaux.

4 Juan Bautista Alberdo était juriste et député dans les premières années de la Confédération argentine. Ses travaux ont inspiré la Constitution nationale de 1853. Domingo Faustino Sarmiento était homme de lettres, militaire et homme politique. Il exerça la présidence de la Nation de 1868 à 1874. Fortement inspiré par les réformes politiques et la philosophie des États-Unis d’Amérique du Nord, il est considéré comme le « père de l’Éducation » en Argentine.
}

concernées par les politiques actives d'immigration promues par l'État Argentin), apportèrent, pendant les $19^{\mathrm{e}}$ et $20^{\mathrm{e}}$ siècles, des savoirs construits au cours d'expériences infructueuses d'intégration dans un modèle productif qui ne leur accordait pas de place dans leurs pays d'origine.

En dépit de cette approche généraliste et civique de l'éducation populaire (selon les termes de Sarmiento), inspirée des idées révolutionnaires qui en Europe formèrent, à la moitié du 19e siècle, les intellectuels argentins, diverses organisations populaires et philanthropiques créèrent et soutinrent des expériences de formation pratique aux métiers. Les premiers syndicats, groupes anarchistes et socialistes et jusqu'à l'État lui-même (bien que de façon minoritaire) donnèrent naissance à la formation aux métiers essentiellement manuels.

À la fin du 19e siècle et à partir de l'École nationale de Commerce (de niveau secondaire) fut créée l'École technique ${ }^{5}$ qui donnera naissance à la première institution de formation de techniciens dans le pays, sur le modèle des disciplines d'ingénieurs connues à l'époque.

Par ailleurs, au début du 20e siècle, des dirigeants représentant les milieux conservateurs impulsèrent des réformes à l'Éducation nationale, proposant de scinder le système éducatif entre une branche propédeutique (destinée à ceux qui pouvaient terminer leurs études primaires et accéder à l'éducation secondaire et ensuite à l'Université) et une autre, professionnalisante, qui permettait à ceux qui ne parvenaient pas à terminer leurs études obligatoires, de se former aux métiers et travaux manuels, de façon à obtenir au moins une éducation pratique qui leur serait « utile pour la vie » et les éloignerait de «l'oisiveté et des vices ».

Cette réforme entra en vigueur en 1914 pour une durée de deux ans et fut annulée par le gouvernement

\footnotetext{
5 En 1897 est créé un département industriel ensuite appelé École Industrielle Nationale. Les spécialités proposées étaient la mécanique, la chimie, l'électricité, le génie civil et naval. Les horaires consacrés à l'atelier furent portés à $25 \%$ maximum, le reste étant dédié à l'enseignement des sciences fondamentales et appliquées (Tedesco, 1970).
} 
d'Hipolito Yrogoyen, représentant les classes moyennes de la société qui voyaient dans l'éducation générale la possibilité de légitimer une voie d'ascension sociale.

Jusqu'à la crise de 1930, nous sommes dans un pays dont les revenus proviennent de l'exportation de viande et de grain, avec une industrie nationale en développement afin d'approvisionner le marché intérieur mais très loin de couvrir tous les besoins du marché local.

Jusqu'au milieu des années 40, l'enseignement spécifique lié au travail, prodigué par l’État, est toujours très restreint. Selon les dates, sur l'ensemble de la population qui fréquentait les établissements scolaires, seulement $10,6 \%$ se répartissaient au sein de quelques modalités existantes de formation professionnelle (Weinberg 1967). En outre, il existait dans le pays, essentiellement dans les centres urbains ou se concentrait la population immigrante, des cours et expériences de formation aux métiers, liés aux mouvements syndicaux, aux partis de gauche et aux sociétés philanthropiques ; cependant, ils ne constituaient pas une proposition articulée.

\section{Une patrie travailleuse et péroniste (1945-1955)}

L'arrivée de Peron ${ }^{7}$ au gouvernement est le fruit d'une profonde transformation de la société Argentine. La mécanisation des campagnes, l'augmentation de la production industrielle et la consolidation du modèle de substitution des importations vont engendrer un nouveau fait historique : el « Cabecita negra $»^{8}$ (tête noire).

\footnotetext{
${ }^{6}$ Hipolito Yrigoyen fut président de 1816 à 1822 et en 1928, il commença un second mandat qui sera interrompu par le premier coup d'État de notre histoire récente, à l'initiative du général Uriburu. Le parti radical, qui représentait les classes moyennes de la société, le considère comme l'un de ses fondateurs.

7 Juan Domingo Peron, président de 1946 à 1955, et ensuite en 1974 jusqu'à sa mort cette même année, est un des personnages les plus influents de la politique argentine. Créateur du parti « Justicialiste » ou « Mouvement peroniste » comme le définissent ses propres partisans, il développa un modèle d'articulation de l'économie et une politique basée sur le marché interne, la souveraineté nationale et l'intervention des syndicats dans la vie publique.

${ }^{8}$ Nom péjoratif en référence à un oiseau commun dans cette région, donné aux migrants de l'intérieur qui venaient pour travailler dans les industries.
}

Les analyses du marché du travail de cette période permettent de comprendre la dimension de ce phénomène. Entre les années 30 et 40, la population active étant inférieure à cinq millions de personnes, on observe une augmentation d'un million de travailleurs dans le secteur industriel (Palomino et Schvartzer, 1993). Arrive ainsi directement dans l'industrie un cinquième de la force de travail disponible, le nombre de personnes qui migrent depuis les provinces pour exercer des activités liées à l’industrie étant toujours plus élevé.

Si le modèle antérieur fut marqué par l'intégration des immigrés et natifs de la " nation », cette période se caractérise par l'intégration des migrants de l'intérieur (exode rural) au travail salarié et à la consommation.

Le système éducatif fait aussi l'objet de transformations importantes. Une commission pour réguler l'activité d'apprentissage dans les entreprises (dépendant du ministère du Travail) est créée, et dans le cadre du ministère de l’Éducation, naît la Direction nationale de l'Éducation technique, qui gère les écoles industrielles existantes (à cette époque déjà il en existait plusieurs, réparties en divers lieux du territoire national, en plus de celle créée à la fin du $19^{\text {e }}$ siècle) et les cours d'arts et métiers qui donneront naissance à ce que, aujourd'hui, on appelle le niveau de formation professionnelle, destinés aux personnes ayant terminé leurs études primaires (ou bien qui les poursuivaient dans le cas des plus de 14 ans.)

Le gouvernement créa une alternative éducative destinée à ce nouveau sujet historique, les travailleurs, ces derniers accédant difficilement à la voie propédeutique valorisée par les classes moyennes de la société qui résistaient au péronisme. Les écolesusines s'organisèrent (écoles d'alternance qui combinaient un temps de travail et un temps de formation) et vers la fin du deuxième mandat interrompu de Peron, l'Université ouvrière nationale (UON), qui allait poursuivre la formation des travailleurs, naît de l'apprentissage dans l'usine et de l'alternance.

Dès lors, des visions divergentes apparaissent quant aux finalités et résultats de cette alternative. Pour certains, la création d'une voie parallèle impliquait de détourner les travailleurs des intérêts légitimes et légitimés par la société (Weinberg, 1984 ; Tedesco, 
Graphique 1

Structure du système éducatif durant les deux premiers gouvernements péronistes (1946-1955)

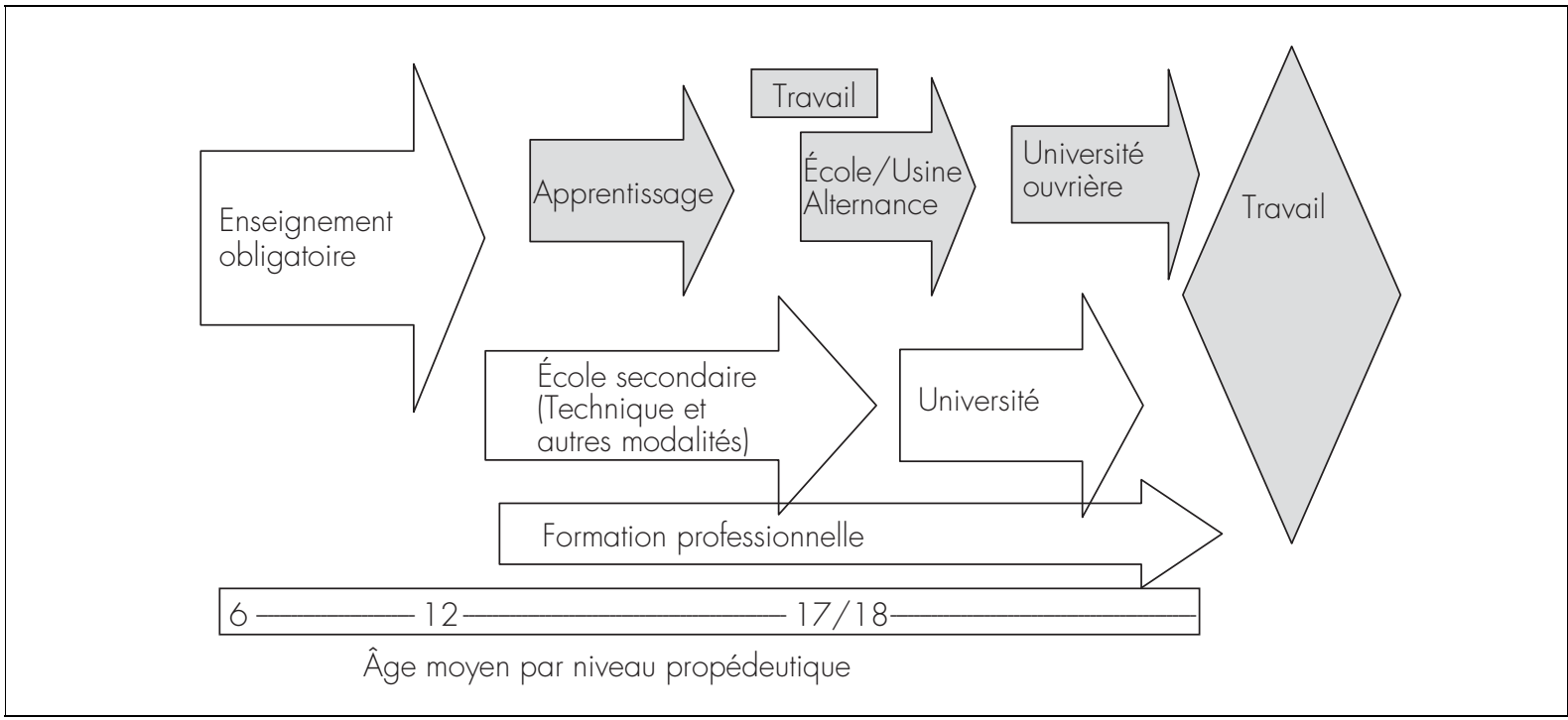

1980); pour d'autres, elle constituait l'alternative viable qui permettait d'intégrer la masse des travailleurs dans un système éducatif qui leur était hostile (Pineau, 1991). Le Graphique 1 montre les diverses voies qui furent élaborées à l'époque.

Même si on note des avancées importantes en matière d'institutionnalisation de la formation professionnelle (représentée non seulement par la voie professionnalisante mais aussi par les modalités techniques et commerciales de la voie propédeutique), elle est encore quantitativement faible si l'on considère l'accroissement exponentiel du marché du travail urbain dans la même période. Les analyses réalisées sur l'évolution de l'enseignement technique et la formation professionnelle dans cette période (Tedesco, 1977 ; Wiñar, 1970 ; Weinberg, 1967) rendent compte de l'insuffisance de leur diffusion en comparaison des autres modalités éducatives existantes.

Quant aux syndicats, conformément à la tradition hétéronome ${ }^{9}$ qui s'inaugure à cette époque, également

\footnotetext{
${ }^{9}$ L'hétéronomie des relations de travail se réfère au règlement des conflits entre capital et travail de façon indirecte, grâce à l'intervention de l'État. Cette position diffère de la relative autonomie que revendiquaient les syndicats anarchistes et socialistes fortement représentés dans les périodes précédentes et aussi dans d'autres pays d'Amérique latine, pour lesquels l'État n'est pas un médiateur mais le représentant du capital.
}

en matière de formation, ils en viennent à jouer un rôle d'interlocuteur de l'État pour la défense de leurs intérêts. L'organisation d'espaces de décision de gestion tripartite (État, syndicats et entreprises) pour tous les thèmes en lien avec la formation s'impose et se poursuit jusqu'à nos jours comme l'expression du système régulateur constitué sous la relation salariale fordiste. Cette action tripartite s'est traduite alors par des accords collectifs de travail (homologués par l'État) et par la gestion quotidienne de ces accords par des commissions internes aux grandes entreprises.

\section{Un pays en développement (1955-1976)}

Le renversement du gouvernement de Peron et la proscription de ce dernier pendant presque vingt ans fit place à une succession de gouvernements élus par le vote populaire et de dictatures militaires; ils soutinrent cependant un même modèle d'accumulation fondé sur la consommation intérieure avec des tentatives de complexification de la production se traduisant par la volonté de développer les moyens de production (Cortez et Marshall, 1991).

Le scénario international, de son côté, (reconstruction européenne après la seconde guerre mondiale et protection des marchés intérieurs des pays respectifs), 
conjugué aux restrictions internes, résultat du manque de compétitivité de la production nationale, limite les potentialités exportatrices de la production nationale, déjà si réticentes devant un marché national capable d'assurer l'accumulation capitaliste locale.

La question du développement, propre à cette époque, et qui connut une grande répercussion en Amérique Latine, apparut avec la théorie du capital humain, l'idée de l'éducation comme facteur de développement.

Ainsi, avec les fragments du sous-système créé par le péronisme pour intégrer les travailleurs, on créa le Conseil national d'éducation technique (CONET) dont dépendront les écoles industrielles de la nation, les centres de formation professionnelle (créés à partir des écoles d'arts et métiers et dans quelques cas dépendant des mêmes écoles techniques); l'Université ouvrière fut transformée en Université technologique nationale afin d'offrir des carrières d'ingénieurs destinées aux sortants d'écoles secondaires, et plus étroitement liées au monde de la gestion et de la production industrielle.

Les réformes opérées dans cette période, aussi bien sur la structure du système éducatif que sur les contenus des programmes d'études, ne connurent pas de grandes modifications jusqu'aux années 90 .

Quant au rôle joué par les syndicats, cette période va constituer une période de fortes tensions. Moment de fractures entre ceux qui assumèrent une position de type collaborationniste avec les différents gouvernements et ceux qui maintinrent une position de lutte et de résistance. Cependant, s'agissant de la formation technique et professionnelle, il n’y eut pas de divergences significatives, l'ensemble des syndicats jouant un rôle minoritaire et relativement influencé par l’État sur la formation professionnelle.

Un élément important et qui est propre à cette période (non par son ampleur mais par rapport à ses effets paradigmatiques) est la création, dans le champ des grandes entreprises industrielles ou de services, d'écoles techniques liées à elles et dans lesquelles les syndicats jouèrent un rôle important. Les firmes Ford (automobile), Olivetti (machines et outils), Philipps (Électronique), Siemens (électronique) et quelques entreprises d'État (Segba électricité) créèrent des écoles de formation de techniciens sur un modèle de gestion mixte.

Dans ces expériences, les entreprises fournirent l'équipement, les espaces de formation pratique (ce qui a toujours constitué un épineux problème au sein des écoles techniques) De même, les entreprises et les syndicats fournirent le personnel enseignant et offrirent aux personnes syndiquées et à leurs enfants les possibilités de formation et d'insertion ultérieurs dans les entreprises. Certes, il s'agissait de grandes entreprises (publiques et privées) dans lesquelles la gestion de la force de travail était fortement régulée. Il en fut tout autrement dans la majorité des entreprises qui généraient de l'emploi, les petites et moyennes, dont les cadres régulateurs furent toujours moins formalisés et où les syndicats eurent moins d'influence directe. Le graphique $\mathbf{2}$ montre la structure du système éducatif de l'époque.

Notons que disparaissent les modalités d'alternance et la formation professionnelle (destinée à la formation dans les métiers de la population suivant la scolarité obligatoire complète, elle ne présente pas d'articulation possible avec le reste des niveaux et modalités).

Selon les données du Centre national de la population, jusqu'en 1960, le taux de scolarisation de niveau intermédiaire était de $9 \%$ et sur la population totale, ceux qui suivaient ou avaient suivi l'enseignement technique représentaient à peine $2 \%$.

En guise d'indicateur de l'évolution historique des modalités technico-professionnelles (essentiellement du niveau moyen), le tableau 1 montre la répartition des élèves par modalité d'enseignement dans les différentes périodes.

Notons que les effectifs des modalités exclusivement propédeutiques (comme c’est le cas du baccalauréat) se stabilisent ou bien augmentent légèrement. La modalité «Normal » (éducation secondaire qui en outre procure le titre de maître du primaire) disparaît, la formation des enseignants se déplace au niveau de l'enseignement supérieur; la part de la modalité commerciale augmente fortement dans l'ensemble.

Sans doute, les transformations qui se sont opérées dans la structure de l'emploi (augmentation du secteur des métiers de la production) ont-elles 
Graphique 2

Structure du système éducatif depuis le développement jusqu'à la loi fédérale d'éducation (1955-1993)

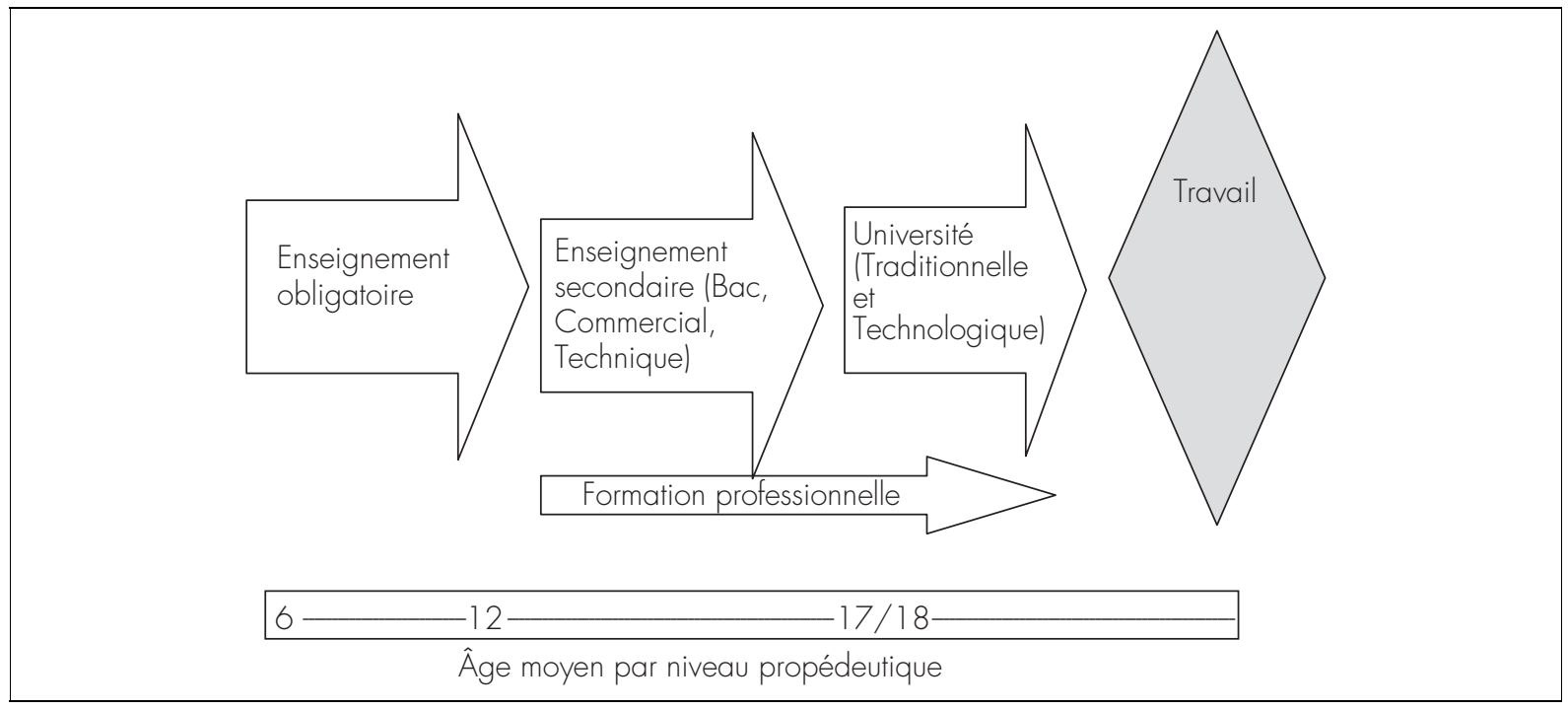

Tableau 1

Effectifs de l'enseignement intermédiaire par modalité en \%

\begin{tabular}{|c|c|c|c|c|c|c|}
\hline & Bac & Normal & Commercial & Industriel & Autres & Total \\
\hline 1930 & 36,2 & 27,4 & 10,2 & 7,3 & 18,9 & 100 \\
\hline 1935 & 39,1 & 23,2 & 10,5 & 8,8 & 18,4 & 100 \\
\hline 1940 & 30,4 & 29,7 & 12,3 & 10,7 & 16,9 & 100 \\
\hline 1945 & 30,9 & 25,0 & 13,9 & 13,3 & 16,9 & 100 \\
\hline 1955 & 23,5 & 20,6 & 17,6 & 18,3 & 20,0 & 100 \\
\hline 1965 & 22,6 & 23,4 & 22,7 & 14,4 & 16,9 & 100 \\
\hline 1970 & 41,4 & 0,1 & 27,8 & 15,2 & 15,6 & 100 \\
\hline 1973 & 37,5 & 0,0 & 31,6 & 16,5 & 14,4 & 100 \\
\hline 1980 & 38,6 & 0,0 & 32,7 & 23,7 & 5,0 & 100 \\
\hline 1984 & 43,5 & 0,0 & 29,6 & 20,3 & 6,6 & 100 \\
\hline 1996 & 44,3 & 0,0 & 28,3 & 17,1 & 10,3 & 100 \\
\hline
\end{tabular}

Source : Élaboré par les auteurs à partir des statistiques du ministère de l’Éducation et de la Culture.

modulé les possibilités de choix de la population dans l'offre éducative.

En 1976, la dictature militaire réorienta le modèle d'accumulation vers l'exportation de produits agricoles et des produits de consommation courante, avec une politique monétaire qui favorisa l'importation de produits manufacturés et la perte de capacité productive de l'industrie nationale qui s'ensuivit; cependant, le marché de l'emploi put amortir l'impact de ces politiques grâce à une pratique répandue en Argentine, le travail indépendant ${ }^{10}$ de cuentrapropismo et de métiers qui ont servi de refuge dans les périodes de crise de l'emploi. Ainsi, le taux de chômage de ces années, quoique élevé (autour des 8 \%) n'entraîna pas une crise comme celle qui allait

${ }^{10}$ Travail indépendant des techniciens et travailleurs des métiers, avec un fort contenu technique ou de services spécialisés. 
survenir au cours de la décennie suivante (Palomino, Schvartzer, 1993).

Dans ce contexte, le système éducatif put maintenir sa structure et conserva l'organisation des contenus qui résultait du processus décrit précédemment. Notamment en ce qui concerne la formation professionnelle et technique, la dictature essaya sans succès, dans les années 80, d'instaurer un plan dual à « l'allemande » motivé sans doute davantage par l'esprit germanophile des chefs militaires que par l'analyse de la réalité productive et sociale du pays. Même si cette expérience eut une certaine longévité en raison de la bureaucratie du système éducatif, elle n'eut pas d'influence majeure ni ne fut capable de modifier les caractéristiques instaurées à la création du CONET (Conseil national d'éducation technique). La situation n'évolua pas durant le premier gouvernement élu par le vote populaire, et va s'amplifier après la crise hyper inflationniste de 1989, et avec le gouvernement de Menen, quand se produisirent de profondes modifications dans la structure du système éducatif et dans la formation technique et professionnelle.

Avant d'aborder ce point, il convient de signaler les problèmes soulevés alors par quelques travaux par rapport à la formation technique et professionnelle.

Le premier élément problématique était lié à l'existence de filières de l'enseignement dédiées à l'industrie dans un pays qui se définissait fondamentalement comme agro-exportateur.

Dans le même temps, les caractéristiques de la production agricole et d'extraction en matière d'usage extensif de la main-d'œuvre ne pouvaient se substituer à l'emploi industriel. Bien que des modalités destinées à la formation de la force de travail rurale aient été créées, elles visaient les techniciens et leur nombre est toujours faible $(1,3 \%$ en 1984 et 2,6 \% en 1996 sur la totalité de la population qui suit des études secondaires).

Un autre problème de nature pédagogique, non résolu par la formation technique professionnelle, était lié à la séparation entre la théorie et la pratique (Testa, 1988 a et b). Tributaire de la division du travail entre conception et exécution, et de son empreinte "ingénieuriale », l'éducation technique professionnelle avait séparé la classe de l'atelier.
La formation professionnelle existante s'appuyait sur la transmission automatique d'opérations et d'instructions avec de faibles références conceptuelles qui auraient permis d'ancrer ces pratiques dans leurs fondements.

Un autre élément qui s'intensifia avec la crise fut l'auto centrage excessif de la FTP et de ses enseignants. Le lien fructueux entre les caractéristiques du travail industriel et la formation était davantage dû à l'expérience personnelle des acteurs (enseignants qui travaillaient dans l'industrie) qu'à une planification et une étude systématiques. Par conséquent, les crises successives de l'emploi et la fermeture de nombreux établissements industriels firent perdre ce lien concrétisé dans l'expérience même des enseignants (Testa, 1988a; Spinosa, 2004b).

Enfin, vers la fin de cette période, on note la volonté de distinguer la formation généraliste du niveau moyen par la création de bacs spécialisés (en sciences, communication, sport, etc.) Par ailleurs, est créée une modalité d'enseignement secondaire pour les adultes. Elle permet la poursuite d'études à ceux qui ne l'avaient pas entamée ou qui avaient abandonné, mais avec un nombre d'années réduit par rapport à la modalité « Normal »; de plus, une part importante de la formation est consacrée à la formation spécialisée dans un champ professionnel, en lien avec l'hypothèse que l'expérience de travail construit des savoirs qui peuvent être utilisés dans la formation générale. Cette modalité, toujours en vigueur de nos jours, n’a jamais été considérée comme une formation alternative; au contraire, elle est considérée comme une formation de moindre niveau académique, destinée aux adultes des classes populaires, même si elle n'a pas encore réussi à transformer en ressources pédagogiques concrètes les hypothèses qui la sous-tendaient.

Un autre élément à retenir est la faible relation entre le nombre des élèves dans l'éducation secondaire, et notamment dans l'enseignement professionnel, et la taille de la population active. Ainsi, si l'accès au marché du travail n'exige pas de formation spécialisée, on peut supposer qu'une action de formation, par le biais de l'entreprise, s'est développée au cours de la vie professionnelle. 


\section{LES PROBLÈMES NON RÉSOLUS : 1990-2001}

\section{L'éducation pour une nouvelle population active sans emploi}

Le premier élément à signaler est le transfert des services éducatifs aux provinces en 1992. Sous couvert de décentralisation dans la gestion et d'efficience administrative - motivée principalement par la nécessité de réduire la dépense publique nationale, conformément à l'injonction des organismes internationaux de crédit aux prises avec la crise de la dette les écoles techniques ${ }^{11}$ et de formation professionnelles, soutenues par le Budget national et dépendant d'un organisme centralisé et unique, devinrent dépendantes tant du point de vue économique qu'en matière de contenu d'enseignement, de la gestion de chacune des provinces et de la ville de Buenos Aires.

Jamais auparavant, à l'exception de quelques expériences, les ministères de l'Éducation des provinces, ni les professionnels, n'avaient pris en charge la formation liée au travail, l'éducation des techniciens et les carences en matière de formation professionnelle relevant essentiellement d'une politique nationale. Ceci engendra une crise et une absence de planification dans la mesure où il n'existait pas, dans les provinces, de cadres techniques et professionnels ayant de l'expérience dans ce domaine.

L’année suivante la promulgation d'une nouvelle loi sur l'éducation apparut comme le résultat d'un processus incertain et avorté de refondation du système éducatif initié par le gouvernement antérieur, exprimé au congrès pédagogique national de $1984^{12}$.

${ }^{11}$ On décentralisa non seulement ces modalités mais aussi les écoles de formation primaire et le reste des modalités d'éducation secondaire et supérieur, qui dépendaient de la Nation.

${ }^{12}$ Imitant le congrès pédagogique impulsé en 1882 par DF Sarmiento, le gouvernement démocratique qui suivit la chute de la dictature organisa un congrès avec une large participation populaire, pour définir une nouvelle éducation pour le pays. Le manque de proposition pour surmonter les problèmes auxquels était confronté le système éducatif, de même que l'absence d'orientation dans ce processus de délibération et de consultation firent que le congrès finit par se réduire à une assemblée de lobbiyistes favorables aux intérêts particuliers. L'église catholique, avec sa forte influence sur l'éducation publique, l'éducation privée en général, certains secteurs du syndicalisme enseignant ou quelques groupuscules politiques partisans affirmèrent leurs prérogatives sans parvenir à une solution, réduisant ainsi toute participation massive dans les décisions.
La «Loi fédérale d'éducation » articule pour la première fois l'ensemble du système éducatif et modifie sa structure et ses contenus. S'instaure ainsi une éducation générale de base de 9 ans obligatoires, et une éducation appelée « Polymodale » qui remplace l'éducation secondaire dans ses diverses modalités.

Cette éducation polymodale a eu pour caractéristique de transformer toute l'éducation post-obligatoire, d'une durée de trois ans, en une éducation liée au monde du travail.

À cet effet, au sein de l'éducation polymodale, cinq orientations furent définies: sciences humaines et sociales, sciences de base (chimie, physique, mathématiques) et naturelles; communications et art, production de biens et services et gestion d'organisations. Ces cinq options comportaient une formation générale commune et une partie spécialisée conformément aux particularités de chaque modalité. La différence, indépendamment du changement de structure et de dénomination, était la possibilité pour chaque institution éducative de créer des espaces de formation plus spécifiques, appelés « Trajectoires techniques et professionnelles » (TTP) qui permettaient une meilleure spécificité des contenus, les assimilant à la formation professionnelle-occupationnelle. Le graphique 3 présente la structure proposée par la Loi fédérale d'éducation.

Le caractère exclusivement propédeutique du bac disparaît, tous les élèves qui, conjointement au polymodal, avaient suivi une trajectoire technicoprofessionnelle pouvaient accéder à la qualification de techniciens ( $\mathrm{A}+\mathrm{B}+\mathrm{C}$ sur le graphique) et ceux qui ne l'avaient pas fait, au-delà de la modalité, obtenaient une certification qui leur permettait de poursuivre des études supérieures $(A+B)$. De même, la formation professionnelle (A + D sur le graphique) demeura une modalité séparée du reste et faiblement représentée (destinée aux plus pauvres) permettant en outre aux trajectoires technico-professionnelles d'être une sorte de formation spécifique destinée à la population ne poursuivant pas d'études secondaires ou qui les avait abandonnées (la trajectoire $\mathrm{A}+\mathrm{C}$ ). Cette possibilité, qui s'ajoutait à la formation professionnelle, bien qu'elle soit également séparée, n’est pas parvenue à se développer. Par exemple, selon les chiffres du recensement de la population de 2001, les 
Structure du système éducatif instauré par la loi fédérale d'éducation (1993-2007)

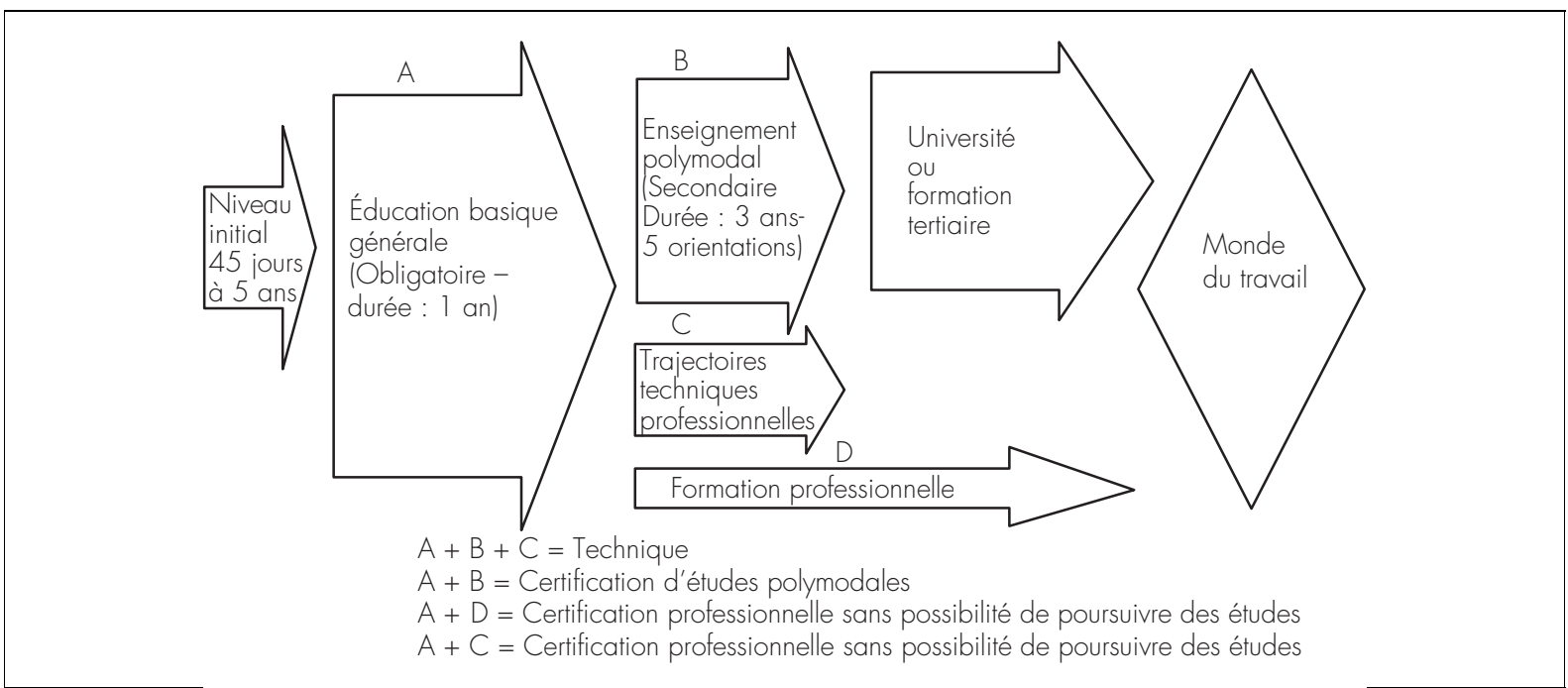

élèves de la formation professionnelle représentent $2 \%$ des plus de 25 ans sans éducation secondaire et $2,28 \%$ de la population active.

Par ailleurs, les stages furent réglementés comme modalité éducative, et l'on introduisit le concept de « compétences " $^{13}$ avec ses implications en termes de formation, certification et conception institutionnelle.

Au cours de la même période, en arguant de la nécessité de modifier les qualifications de la force de travail dans une période de restructuration productive, les organismes internationaux vont impulser des programmes de formation qui dépendront d'abord du ministère de l'Économie et ensuite du Travail. Lesdits programmes, similaires dans l'ensemble des pays de la région, étaient liés aux politiques actives de l'emploi et se sont développés dans le cadre d'un marché ouvert d'offres et de demandes de formation, dans lequel l'organisme responsable faisait office d'intermédiaire.

\footnotetext{
${ }^{13}$ Nous ne ferons pas référence ici au débat existant autour du concept de compétences. Nous mentionnerons simplement, dans la lignée de ce qui a été développé par Lucy Tanguy (2001), le caractère idéologique et réducteur de ce terme transposé dans le champ de la formation. Le terme " compétence », introduit par les organismes internationaux de crédit qui financèrent la réforme, conjugué à l'adhésion des organismes éducatifs de l'époque témoignaient d'une acceptation acritique non seulement du concept lui-même mais aussi de celui que nous appellerons le " programme compétences », qui comprend outre une orientation éducative pragmatique et behavioriste, une forme de précarisation de l'emploi et de contournement de la négociation collective en matière de classification professionnelle (Spinosa, 2005).
}

Ces propositions de formation professionnelle, au-delà des nombreuses critiques qu'elles ont suscitées quant à leur manque d'adéquation aux besoins particuliers, le rapport défavorable entre coûts et bénéfices en termes d'augmentation de l'emploi, jusqu'au fait qu'elles ont favorisé des formes d'emploi précaire, par le biais des stages, séparées explicitement du reste du système éducatif, ne permettaient pas la poursuite d'études. Bien qu'essentiellement destinées aux personnes ne possédant pas le niveau secondaire complet, elles ne leur permettaient pas d'obtenir une certification ayant de la valeur dans le système éducatif (et comme nous l'avons vu, pas davantage sur le marché du travail).

Contrairement aux anticipations des politiques, l'augmentation du niveau de scolarisation de la population (dans tous les niveaux) et la sur-certification des jeunes de milieux défavorisés (par les programmes évoqués dans le paragraphe précédent) n’induisent pas la diminution des taux du chômage, mais le développement de phénomènes comme la sur-éducation (Perez P., 2005).

La crise politique, économique et financière que traversa l'Argentine, au début des années 2000²,

\footnotetext{
${ }^{14}$ En 2001, après avoir mis en place des mesures pour retenir l'épargne de la population dans le système financier, le président élu F. De la Rua renonça et quatre présidents se succédèrent au cours des dix années qui suivirent. Les classes moyennes et autres mouvements populaires criaient "qu'ils s'en aillent tous", illustrant clairement la crise de la représentativité.
} 
engendra un questionnement sur tout ce qui s'était produit dans la période antérieure en matière de politique publique, du rôle de l'État et des organisations dans la société.

Il en résultat une forte désarticulation sociale, avec plus de la moitié de la population sous le seuil de pauvreté, un chômage dépassant 20 \% de la population active et une économie dévastée par la fuite des capitaux.

En matière éducative, la situation était très hétérogène. Au démantèlement du système d'éducation nationale (comme produit d'une inégale mise en œuvre du modèle éducatif dans chaque province illustrant l'inégalité préexistante et le transfert produit), s'ajoutèrent le manque d'investissement public et la carence d'un modèle commun articulant les différents niveaux et modalités de la formation. (Balduzzi J., 2001 ; Feldfeber M. et Ivanier A., 2003 ; Testa J., Sanchez P., 2003).

La formation professionnelle restait marginale dans le système éducatif, sans lien avec le reste des modalités, caractérisée par le manque de ressources, la faible formation de ses enseignants et s'adressait uniquement aux classes les plus pauvres de la société pour des activités très informelles. La tentative de professionnaliser l'ensemble de l'éducation moyenne ne se concrétisa pas dans la pratique quotidienne des écoles. Les élèves diplômés du niveau secondaire optaient, dans la mesure de leurs possibilités, pour l'éducation supérieure (Testa, 1998), et sur un marché du travail fortement précarisé et restreint, avec une sur-offre de personnel qualifié, il ne semblait pas y avoir de place pour les diplômés de l'éducation Polymodale, excepté dans les emplois dénommés «emplois jeunes », caractérisés par une faible qualification, une forte rotation de personnel et la précarité (emplois dans les hypermarchés, Mac Donald, etc.)

Comme nous l'avons développé dans d'autres travaux à partir de l'analyse exhaustive de quelques modalités éducatives (Spinosa, 2004), la réforme proposée dans les années 90 semblait être portée par une idée de marché du travail et d'organisation des processus productifs correspondant uniquement à un secteur minoritaire des entreprises, celles dont l'intensité capitalistique est élevée et le nombre d'emplois faible. L'hypothèse selon laquelle ce modèle allait se diffuser et se généraliser à l'ensemble du tissu productif constitua une grave erreur de lecture des principales variables économiques et politiques du pays. Sans oublier de mentionner le manque de questionnement d'un modèle de société d'exclusion, d'un marché du travail paupérisé et précaire, avec une polarisation excessive des qualifications, marché au service duquel (et sous la peur du chômage qui le caractérisait) s'organisèrent les réformes et les modèles proposés.

\section{Vers un modèle viable : Crises et contre-réforme éducative : 2001 et suivantes}

Les périodes de conflit social important permettent souvent de modifier les espaces de négociation et la façon d'analyser les problèmes pour lesquels il semblait n'exister qu'une solution.

La période initiée sous la présidence de Kirchner (la modification du taux de change et l'accroissement des taxes aux exportations de produits de consommation courante) a rendu possible un repositionnement des acteurs sociaux auparavant écartés, et un lent processus de ré-articulation de l'appareil productif non dépourvu de conflits ${ }^{15}$.

Au-delà des faits, la transformation radicale s'est produite dans le discours public. Le rôle de l'État dans la régulation de l'économie, le retour à un modèle de pays avec une industrie et de l'emploi, la nécessité de " retrouver la culture du travail », sont les prémisses intégrées aux agendas politiques.

Dans ce scénario, l'éducation a commencé à apparaître comme la dépositaire d'un mandat social, destinée à contribuer au développement de l'économie et des individus.

Vers la fin 2005 fut instaurée une nouvelle législation sur l'éducation technique et professionnelle (Loi 26058) qui essaya, au niveau national, de remettre au

15 En2008, le conflit pour le modèle économique connut un pic de tension quand le gouvernement tenta de modifier le régime des taxes à l'exportation de soja. Cette mesure conduisit à la manifestation publique des secteurs les plus concentrés de l'économie et des classes moyennes contre le péronisme, en faveur d'un changement de modèle économique. 
centre la conception et la définition des politiques du secteur. Cette réglementation se proposait de revoir la séparation entre les diverses modalités et juridictions.

Les modalités et niveaux existants furent maintenus, conformément à la législation générale sur l'éducation (loi Fédérale d’Éducation).

Fin 2007, suite à la consultation des différents acteurs et organismes, fut promulguée une nouvelle loi d’Éducation nationale qui remplace la loi Fédérale de 1993. Globalement, cette réglementation place la formation technique professionnelle sous les critères définis auparavant et modifie la structure du système éducatif, le transformant en une éducation primaire de 6 ou 7 ans (de l'âge de 6 ans jusqu'à 11 ou 12 ans ; ceci étant défini par chaque juridiction) et une éducation secondaire de 5 ou 6 ans (de l'âge de 12 ou 13 ans jusqu'à 17 ou 18 ans), rétablissant ainsi l'unité du cycle secondaire et renversant d'une certaine façon la situation engendrée par la création du polymodal. Les deux niveaux sont obligatoires, puisque l'obligation est portée à 12 ans au total.

Même si le travail est un des piliers sur lesquels s'appuient les finalités de l'éducation, et que l'éducation secondaire obligatoire représente le prolongement de la formation pour le travail, il n'y a pas eu globalement d'avancées significatives en matière d'articulation des instances de formation spécifique à la formation générale.

Ainsi, la formation secondaire comporte, dans toutes ses modalités, (et particulièrement dans le cas de la modalité technico-professionnelle) à la fois des objectifs propédeutiques et professionnalisants, tandis que la formation professionnelle spécialisée n’a pas été expressément envisagée, étant une fois de plus reléguée et dispersée à côté d'autres expériences informelles ou organisées par des institutions particulières.

Parallèlement à cette réorganisation de l'Éducation nationale, le ministère du Travail, les centrales syndicales, les organismes chargés du développement et les ministères de l’Éducation des juridictions ont proposé la création de domaines, organismes ou programmes qui articulent l'éducation et le travail. Ces tentatives d'institutionnalisation de la relation entre éducation et travail ont fonctionné jusqu'à présent comme des espaces de discussion, de propo- sitions et d'expression des revendications sectorielles (syndicats, entreprises, enseignants) sans réussir encore à modifier le mode de fonctionnement des organisations éducatives ni leurs contenus, dans lesquelles survivent les critères et pratiques historiquement construits ${ }^{16}$.

Notons que l'origine de chacune de ces actions définit fortement le caractère et la façon d'appréhender la relation. Si les actions sont à l'initiative des ministères de l'Éducation, elles tendent à placer l'école et la formation scolaire traditionnelle au centre, laissant de côté, ou envisageant avec beaucoup de difficulté d'autres formes de construction et de transmission du savoir qui ne passent pas par les salles de classe. Cela suppose en outre une plus grande valorisation de la théorie par rapport à la pratique et en général une dévalorisation du travail manuel.

Si à l'inverse, les actions sont proposées par les acteurs liés au travail, que ce soit le ministère du Travail ou les organismes employeurs, l'accent est alors mis sur la formation spécialisée, la capacité d'influer à court terme sur les problèmes de la productivité ou de l'emploi. Au nom de principes idéologiques comme « il faut changer d'emploi et de profession tout au long de la vie » ou « l'emploi à vie c'est fini » ou bien à partir de la généralisation d'une modalité technologique qui est loin d'être homogène, comme en attestent les études de cas en entreprises (Texta, Figari, Spinosa, 2001), la formation est dissociée des trajectoires éducatives plus générales des individus.

Les syndicats en général, selon leur profil corporatiste (De La Garza Toledo, 2001), adoptent l'une ou l'autre des positions (formation plus scolarisé ou plus spécialisée) selon l'origine des ressources.

\footnotetext{
16 À titre d'illustration, nous mentionnerons dans le cas de la Province de Buenos Aires, la création d'une Direction chargée de l'éducation et du travail, d'une agence de certification des savoirs du travail et de l'impulsion à la création de conseils qui réunissent les différents acteurs impliqués dans la thématique. À Buenos Aires se sont ainsi développés des programmes et actions tendant à reconnaître cette thématique, de même que dans d'autres provinces.
} 
En analysant les aléas rencontrés dans la construction du système éducatif et son rapport à l'organisation d'un modèle d'économie nationale, on pourrait conclure qu'il n'existe toujours pas de forme d'organisation éducative qui contribuerait massivement à la formation de la force de travail. Cependant, tant à l'époque de la substitution des importations qu'à l'heure actuelle (toutes deux centrées sur l'accumulation à partir du marché intérieur), la rapide intégration des personnes sans emploi ou occupées sur des emplois marginaux, dans le secteur industriel, rend compte d'un processus de formation important. Dans les deux cas, ce processus ne peut être considéré comme le fruit de l'action volontariste du système éducatif mais celui de l'intervention d'autres acteurs dans la construction des savoirs spécifiques afin de faire face aux besoins de la production.

Ainsi, l'insertion professionnelle, la productivité et l'efficacité des interventions professionnelles paraissent dépendre davantage des conditions structurelles de fonctionnement de l'économie, des décisions des entreprises en matière d'organisation du travail et des régulations que l'État impose au marché du travail, que des conditions subjectives de la force de travail (ses savoirs, ses ressources identitaires, etc.), pour l'accès à l'emploi.

Cela n'enlève rien à la valeur de l'éducation dans la construction de connaissances et de capacités des travailleurs ni à la configuration de trajectoires qualifiantes, mais la planification exige une analyse qui prend en compte les différents niveaux d'analyse dans lesquels se joue la relation entre éducation et travail, de même que les dimensions qu'elle englobe.

La carence d'une structure articulée au niveau organisationnel pour la formation spécialisée a conduit dans de nombreux cas à penser que cette carence est responsable des problèmes de productivité et de chômage. En conséquence, nous pensons que la solution doit consister en la mise en place d'un système articulée et d'organisations spécifiques de formation pour le travail. Souvent, les propositions formulées ont occulté l'existence de conditions structurelles contraires à la création d'emplois et de pratiques qualifiantes dans l'espace de travail ; de la même façon qu'a été occulté l'impact sur les trajectoires particulières des personnes du passage par d'innom- brables expériences de formation spécifique sans articulation entre elles ou assujetties aux besoins conjoncturels de l'emploi dans un secteur donné.

Tel a été le cas d'une grande partie des actions engagées dans les années 90 qui, en considérant la formation pour le travail comme résultant uniquement de l'action de l'État, oublieuses de l'histoire particulière de l'exercice de cette fonction en Argentine dans laquelle d'autres acteurs jouèrent un rôle majeur, voulurent imposer un modèle impliquant la destruction du modèle existant sans réussir à en imposer un nouveau.

Le bref historique présenté permet d'appréhender la façon dont les politiques basées sur la conjoncture ont fluctué entre la promotion de la formation spécifique comme moyen de contribuer à la transformation du modèle d'accumulation ou bien, à partir de la position contraire, comment l'accent a été mis sur la formation générale, en négligeant la formation pratique de la force de travail.

Les relations sociales engagées par les différents acteurs, et en particulier entre l'État et les syndicats, ont contribué à une lutte pour le contrôle des appareils étatiques chargés de la formation pour le travail, ce qui s'est traduit historiquement par la confrontation entre les ministères de l'Éducation et du Travail pour le contrôle de la formation technique et professionnelle et le rôle qui y est joué par les représentants syndicaux. De son côté, le secteur entrepreneurial, dont la représentation est scindée entre les associations liées aux grandes entreprises et les nombreuses autres entités qui tentent d'absorber les petites et moyennes entreprises, réclament automatiquement et systématiquement une adéquation de l'éducation aux besoins de recrutement de main-d'œuvre, en mentionnant dans leurs critiques envers l'éducation, l'obsolescence des équipements ou encore le manque d'aptitudes spécifiques ${ }^{17}$.

\footnotetext{
17 Des travaux réalisés dans le secteur chimique nous ont permis de vérifier l'excessive idéologisation de ce discours, et la forte divergence entre les responsables des ressources humaines et les responsables de la production en matière de valorisation de la formation apportée par le système éducatif dans ladite spécialité (Testa, Figari, Spinosa, 2003). De même, des travaux réalisés dans de grandes entreprises montrent aussi le décalage entre ce type de demandes génériques et les pratiques effectives de formation dans les entreprises (Gentili, 1994).
} 
La façon de concevoir la production de connaissance et son utilisation par les individus a généré des actions divergentes qui supposent d'un côté, la primauté de l'éducation disciplinaire, théorique, faisant de la pratique un champ d'application et d'un autre côté, le primat de l'expérience, encourageant des formes rapides d'insertion et d'expérimentation, sans lieu défini pour la reconstruction théorique. Ceci constitue sans doute un aspect fondamental pour l'organisation des actions et la compréhension des mécanismes d'articulation entre les dimensions productives et formatives des organisations sociales et des individus. La possibilité de construire des itinéraires (en termes de chemins possibles) conduisant à des formes différentes de construction des différents types de savoirs implique une profonde transformation des principes sur lesquels se construit la structure scolaire (au-delà des niveaux et modalités), ce qui est encore à l'état embryonnaire en Argentine tant du point de vue de la recherche académique que des politiques publiques (Spinosa, 2006 et 2007 ; Spinosa et Drolas, 2009).

Au-delà des tentatives actuelles pour redéfinir la formation professionnelle et technique, et des ressources financières et institutionnelles destinées à son développement, les avancées sont encore faibles en matière de construction d'une politique publique qui englobe et régule l'ensemble des acteurs intervenant dans l'articulation entre formation générale et spécifique, et qui envisage l'impact que cela a sur les trajectoires éducatives et professionnelles des personnes.

Même si la cause de cette difficulté doit être recherchée dans la précarité des conditions structurelles (dans le sens de définitions de long terme sur le modèle d'accumulation), nous pensons que le manque d'outils conceptuels qui rendraient possible une analyse complexe et intégrée de la façon dont se résout la relation éducation-travail en Argentine, contribue à l'adoption de mesures partielles qui occultent les responsabilités historiques qu'à par exemple eu le secteur productif dans la formation de la force de travail, ou bien l'impact du développement de certifications issues de la multiplication des offres en matière de formation spécifique sur sa valorisation sur le marché du travail.

$\mathrm{Au}$ centre de ces débats non résolus, les individus, travailleurs et travailleuses, tentent de construire, au travers de leurs trajectoires singulières et collectives, la façon d'habiter une Argentine toujours en devenir.

Traduction de l'espagnol au français : Mireille Zangani (Céreq), avec l'aimable collaboration des auteurs.

\section{Bibliographie}

Balduzzi J. (2001), “Desigualdad y exclusión educativa en el 'tercer ciclo' de la provincia de buenos aires”. Cuadernos de información e investigaciones. SUTEBA. Buenos Aires.

Carton M. (1985), La educación y el mundo del Trabajo. UNESCO.

Cortez R. y Marshall A. (1991), "Estrategias económicas, intervención social del estado y regulación de la fuerza de trabajo. Argentina 1880-1990”, En Estudios del Trabajo $n^{\circ} 1$. ASET, Buenos Aires.
De La Garza Toledo E. (2001), "Las transiciones políticas en América Latina, Entre el corporativismo sindical y la pérdida de imaginarios colectivos” En De La Garza Toledo E. (comp.), Los sindicatos frente a los procesos de transición política, Clacso, Buenos Aires.

Feldfeber M., Ivanier A. (2003), "La descentralizacion educativa en argentina: el proceso de transferencia de las instituciones de formacion docente", Revista Mexicana de Investigación Educativa mayoagosto 2003, vol. 8, n 18 pp. 421-445. 
Gentili P. (1994), Poder Económico, Ideología y Educación, Miño y Davila Editores, Buenos Aires, p. 287.

Palomino H., Schvartzer J. (1993) "Del pleno empleo al colapso” En Revista Encrucijadas. UBA, Buenos Aires.

Perez P. (2005), Sobre educación en el mercado de trabajo argentino en un período de desempleo masivo (1995-2003). Presentación al Séptimo Congreso Nacional de Estudios del Trabajo. ASET, Buenos Aires.

Pineau P. (1991), Sindicatos, estado y educación técnica (1936-1968), CEAL Buenos Aires.

Spinosa M. (2004), "El conocimiento en la conformación de identidades profesionales ¿Los técnicos, una especie en vías de extinción?” En Battistini O. (comp.) El trabajo frente al espejo. Prometeo, Buenos Aires.

Spinosa M. (2004), La transformación de la Educación Técnica: contradicciones, debilidades y resultados de un análisis sesgado del mundo del trabajo. Presentacion al Congreso Internacional: La responsabilidad social de la Sociología de la Educación frente a lo nuevos Movimientos Sociales emergentes”. 25 al 28 de agosto, FFyL. U Buenos Aires.

Spinosa M. (2005), “Del 'saber' al 'saber ser'. Las calificaciones en el nuevo escenario de las relaciones de trabajo” En Fernández A. (Comp.) Estado y Relaciones Laborales. Prometeo, Buenos Aires.

Spinosa M (2006), "Los Saberes del Trabajo. Ensayo sobre una articulación Posible” En Revista Anales de la educación Común. Tercer siglo año 2, nº 4.

Spinosa M. (2007), El reconocimiento de los saberes del trabajo. O de cuando se usan lupas para mirar las estrellas. Cd del congreso latinoamericano de sociología del trabajo. ALAST, Montevideo.

Spinosa M. y Drolas A. (2009), "De la certificaciónformación a la certificación-aprendizaje en el proceso de integración del Mercosur. ¿Qué conse- cuencias para la construcción de las trayectorias profesionales?” Calificaciones y Empleo ${ }^{\circ} 62$ Piette / Cereq.

Tanguy L. (2001), "De la Evaluación de los puestos de trabajo a la de las cualidades de los trabajadores. Definiciones y usos de la noción de competencias" En Neffa J.C y De la Garza Toledo E. (Comp.) El trabajo del futuro. El futuro del trabajo. Clacso, Buenos Aires.

Tedesco J.C. (1977), Industrialización y Educación en La Argentina. UNESCO Proyecto "Desarrollo y Educación en América Latina y el Caribe”.

Tedesco J.C. (1980), La educación Argentina. CEAL, Buenos Aires.

Testa J. (1988a), Proyecto pedagógico y organización escolar e institucional. CONET - CONICET, Buenos Aires.

Testa J. (1988b), Una propuesta integral de renovación del ciclo superior de los técnicos electrónicos. CEIL PIETTE, Buenos Aires.

Testa J. (1992), “Aspectos cuantitativos y cualitativos de la oferta de formacion profesional. Caracterizacion de los perfiles profesionales” Informe $n^{\circ} 16$, programa de Asistencia Tecnica. BIRF. PNUD, Buenos Aires.

Testa J. (1998), “Acerca del abordaje del imaginario educativo laboral de los adolescentes de la ciudad de Buenos Aires”. GCBA- CEIL PIETTE - FCSoc U, Buenos Aires.

Testa J., Figari C., Spinosa M. (2001), Cambios en los perfiles profesionales de los técnicos en la industria química. Presentación al $5^{\circ}$ Congreso de ASET, Buenos Aires.

Testa J., Sanchez P. (2003), Una perspectiva crítica y alternativa en relación con la formulación, diseño e implementación de los Trayectos Técnicos Profesionales. Presentación a las Jornadas: A diez años de implementación de la Ley Federal de Educación. 
Disponible en:http://www1.hcdn.gov.ar/dependencias/ceducacion/aportes13.html.

Weinberg P.D. (1967), La enseñanza técnica industrial en la Argentina 1936-1965. Instituto Di TellaCIE, Buenos Aires.
Weinberg G. (1984), Modelos Educativos en la historia de América Latina. Ed. Kapelusz, Buenos Aires.

Wiñar D. (1970), Poder político y educación: el peronismo y la CNAOP, Buenos Aires. ITDT

\title{
Résumé
}

\section{L'enseignement professionnel en Argentine : entre volontarisme et isolement dans la recherche d'un pays possible}

\author{
Martín Spinosa et Julio Testa
}

L'article se propose de revisiter la notion de formation professionnelle et sa construction historique en Argentine, à partir de la multifonctionnalité que cette notion a acquise dans les politiques publiques comme réponse à divers problèmes sociaux. II se propose de naturaliser un discours répandu qui fait reposer sur les organisations éducatives et les individus la responsabilité de former la force de travail pour le développement économique, sans prendre en compte les différents acteurs sociaux qui interviennent dans ce champ. À cet effet, une réflexion est développée sur les périodes historiques de construction du rapport entre éducation et travail selon les différents régimes d'accumulation, pour conclure sur la nécessité de construire des approches larges et intégratrices, appelées compréhensives, afin de rendre compte des divers éléments qui interviennent dans la relation entre éducation et travail.

\section{Mots clés}

Enseignement technique-professionnel, formation professionnelle, système éducatif, Argentine Journal of Economic Literature: I 21 